Available online at http://jddtonline.info

RESEARCH ARTICLE

\title{
SYNTHESIS, CHARACTERIZATION AND BILOGICAL SCREENING OF SOME NOVEL INDOLE BASED 1,2,4-TRIAZOLO 1,3,4-THIADIAZINES
}

\author{
Mahipal Reddy Yata, Raghu Vardhan Reddy Mekala*, Ravi Prasad Talagadadivi \\ Department of Chemistry, Kakatiya University, Warangal, Andhra Pradesh, India \\ *Corresponding Author's E mail: drmrvr@gmail.com
}

\begin{abstract}
S:
A series of novel 3-(1H-indole-4-yl)-6-aryl-7H-[1,2,4]-triazolo-[3,4-b][1,3,4]-thiadiazines (6a-f) were synthesized by involving $1 H$-indole-4-carboxylic acid (1) as raw material and $1 H$-indole-4-carboxylic acid ethyl ester (2), $1 H$-indole-4carboxylic acid hydrazide (3), 5-(1H-indole-4-yl)-[1,3,4]oxadiazole-2-thiol (4) and 4-amino-5-(1H-indol-4-yl)-4H-[1,2,4]triazole-3-thiol (5)as intermediates. The chemical structures of the all newly synthesized compounds were elucidated by their $\mathrm{IR},{ }^{1} \mathrm{H}$ and ${ }^{13} \mathrm{C}$ NMR, mass spectral data and elemental analysis. Further, the target compounds were used to find their antifungal and nematicidal activity.
\end{abstract}

Key-Words: Indole, 1,2,4-Triazole, 1,3,4-Thiadiazines, Antifungal activity, Nematicidal activity.

\section{INTRODUCTION:}

Recently, it was reported that the heterocyclic moiety such as triazole based thiadiazoles and thiadiazines possess variety of pharmacological activities like antimicrobial ${ }^{1}$ antiviral ${ }^{2}$ antibacterial, ${ }^{3}$ antiinflammatory, ${ }^{4}$ herbicidal ${ }^{5}$ and anti-HIV-1. ${ }^{6}$ On the other hand, it has been reported that certain compounds bearing a thiadiazole and 1,2,4-triazole nucleus possess significant anti-inflammatory activity. ${ }^{7}$

These initial reports stimulated us to integrate thiadiazine moiety in triazole frame work, since these systems possess well documented antimicrobial and nematicidal activity. The target compounds, 3-(1H-indole-4-yl)-6aryl-7H-[1,2,4]-triazolo-[3,4- $b][1,3,4]$-thiadiazines (6a-f) have been prepared by using commercially available $1 H$ indole-4-carboxylic acid (1) as raw material and by involving $1 H$-indole-4-carboxylic acid ethyl ester (2), $1 H$-indole-4-carboxylic acid hydrazide (3), 5-( $1 H$-indole4-yl)-[1,3,4]oxadiazole-2-thiol (4) and 4-amino-5-(1Hindol-4-yl)-4H-[1,2,4]-triazole-3-thiol

as intermediates.

The initial intermediate, $1 H$-indole-4-carboxylic acid ethyl ester (2) has been prepared through esterification by boiling of a mixture of $1 \mathrm{H}$-indole-4-carboxylic acid (1) and sulfuric acid in ethanol for $4 \mathrm{~h}$. The compound 2 was reacted with hydrazine hydrate in absolute ethyl alcohol at reflux for $8 \mathrm{~h}$ to get $1 \mathrm{H}$-indole-4-carboxylic acid hydrazide (3). The intermediate, 5-(1H-indole-4-yl)$[1,3,4]$ oxadiazole-2-thiol (4) for the synthesis of title compounds was prepared by the cyclization of compound 3 with carbon disulphide in the presence of potassium hydroxide in ethanol at reflux for $14 \mathrm{~h}$ followed by acidification. Further the compound 4 when reacted with hydrazine hydrate in ethanol at reflux for 6 $\mathrm{h}$ resulted 4-amino-5-(1H-indol-4-yl)-4H-[1,2,4]triazole3-thiol (5). Finally, the compound $\mathbf{5}$ has been condensed successively with a variety of phenacylbromides in ethyl alcohol under reflux for $8-10 \mathrm{~h}$ to get the title compounds, 3-(1H-indole-4-yl)-6-aryl-7H-[1,2,4]triazolo-[3,4- $b][1,3,4]$-thiadiazines (6a-f). The chemical structures of the all newly synthesized compounds were elucidated by their IR, ${ }^{1} \mathrm{H}$ and ${ }^{13} \mathrm{C}$ NMR, mass spectral data and elemental analysis. Further, the target compounds were used to find their antifungal and nematicidal activity.

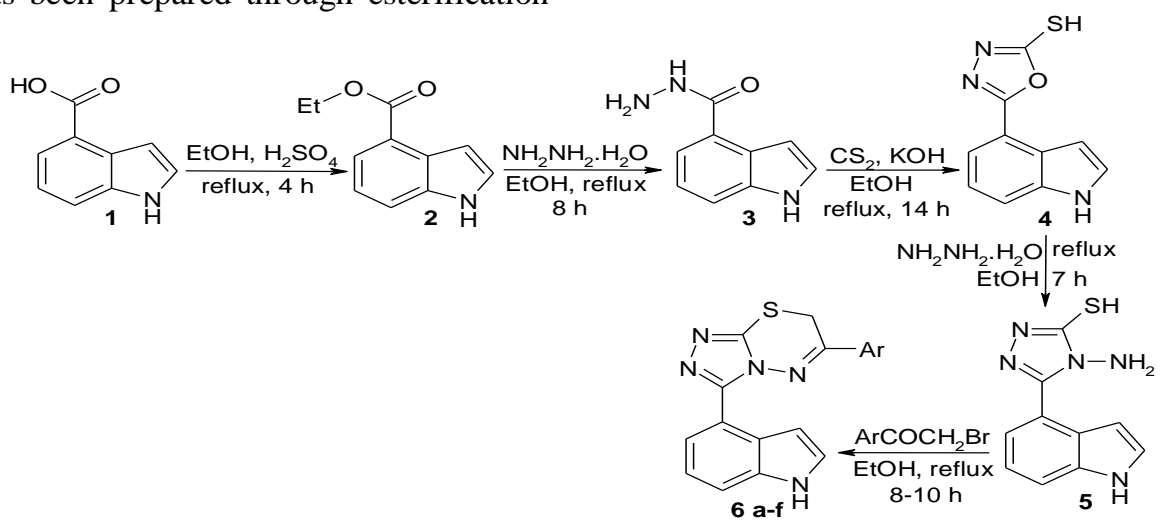

Scheme 1: $6 \mathrm{Ar}=\left(\right.$ a) $\mathrm{C}_{6} \mathrm{H}_{5}$; (b) 4- $\mathrm{OCH}_{3} \mathrm{C}_{6} \mathrm{H}_{4}$; (c) $4-\mathrm{ClC}_{6} \mathrm{H}_{4}$; (d) $4-\mathrm{BrC}_{6} \mathrm{H}_{4}$; (e) 4- $\mathrm{NO}_{2} \mathrm{C}_{6} \mathrm{H}_{4}$; (f) $4-\mathrm{OHC}_{6} \mathrm{H}_{4}$ 


\begin{tabular}{cccrc}
\hline Compound & C. albicans & A. Fumigatus & T. Rubrum & T. Mentropyhte \\
\hline $\mathbf{6 a}$ & 25.0 & 12.5 & $>50.0$ & 25.0 \\
$\mathbf{6 b}$ & 25.0 & 25.0 & 25.0 & 25.0 \\
$\mathbf{6 c}$ & 12.5 & 6.25 & 3.12 & 3.12 \\
$\mathbf{6 d}$ & 25.0 & 12.5 & 6.25 & 12.5 \\
$\mathbf{6 e}$ & 3.12 & 12.5 & 12.5 & 25.0 \\
$\mathbf{6 f}$ & 50.0 & 25.0 & 12.5 & 12.5 \\
Amphotericin B & 6.25 & 3.12 & 3.12 & 3.12 \\
\hline
\end{tabular}

Table 2: Median lethal dose ( $\left.\mathrm{LD}_{50}, \mathrm{ppm}\right)$ of compounds 6a-f

\begin{tabular}{lccccccc}
\hline Nematicide & $\mathbf{6 a}$ & $\mathbf{6 b}$ & $\mathbf{6 c}$ & $\mathbf{6 d}$ & $\mathbf{6 e}$ & $\mathbf{6 f}$ & Levamisole \\
\hline D. myceliophagus & 170 & 270 & 950 & 430 & 570 & 190 & 170 \\
C. elegans & 190 & 220 & 870 & 200 & 610 & 780 & 180
\end{tabular}

\section{ANTIFUNGAL ACTIVITY}

Compounds 6a-f were screened for their antifungal activity against four fungal organisms viz., Candida albicans, Aspergillus fumigatus, Trichophyton rubrum, and Trichophyton mentagrophytes in dimethyl sulfoxide by broth dilution method. ${ }^{[8]}$ The minimum inhibitory concentration (MIC, $\mu \mathrm{g} / \mathrm{mL}$ ) were measured and compared with the standard drug Aamphotericin B (Table 1). Among the screened compounds, 6c is highly active against $T$. rubrum, T. mentagrophytes, $6 \mathbf{e}$ is also active against only $C$. albicans and $\mathbf{6 g}$ is highly active against $C$. albicans, $T$. mentagrophytes and the activity of these compounds are almost equal to the standard. All the compounds in this series exhibited either excellent or moderate activity towards different organisms. None of the compounds is inactive against any one of the organism. It is interesting to note that $\mathbf{6 e}$ showed excellent antifungal activity towards $C$. albicans at the concentration of $3.12 \mu \mathrm{g} / \mathrm{mL}$, which is less than the concentration of the standard.

\section{NEMATICIDAL ACTIVITY}

All the newly synthesized compounds $\mathbf{6}$ a-f in this study were also assayed for their nematicidal activity against Ditylenchus myceliophagus and Caenorhabditis elegans by aqueous in vitro screening technique ${ }^{[9]}$ at various concentrations. The results have been expressed in terms of $\mathrm{LD}_{50}$ i.e. median lethal dose at which $50 \%$ nematodes became immobile (dead), and compared with the standard drug levamisole. The screened data reveal that, 6a is the most effective against $D$. myceliophagus and $C$. elegans with $\mathrm{LD}_{50}$ of 170 and $190 \mathrm{ppm}$, respectively. The compounds $\mathbf{6 d}$ and $\mathbf{6 f}$ are also most active against $C$. elegans with $\mathrm{LD}_{50}$ of $200 \mathrm{ppm}$ and D. myceliophagus with $\mathrm{LD}_{50}$ of $190 \mathrm{ppm}$, respectively. The activity of $\mathbf{6 a}$ is almost equal to the activity of the standard Levamisole. The other tested compounds showed moderate activity. The $\mathrm{LD}_{50}$ values of the compounds screened are presented in Table 2.

\section{EXPERIMENTAL}

(C) 2011, JDDT. All Rights Reserved JDDTAO
All the reagents and solvents were used as purchased without further purification. Melting points were determined on a Fisher-Johns melting point apparatus and are uncorrected. IR spectra were obtained on a Perkin-Elmer BX serried FTIR 5000 spectrometer using $\mathrm{KBr}$ pellet. NMR spectra were recorded on a Varian 300 $\mathrm{MHz}$ spectrometer for ${ }^{1} \mathrm{H}-\mathrm{NMR}$ and $100 \mathrm{MHz}$ for ${ }^{13} \mathrm{C}-$ NMR. The chemical shifts were reported as ppm down field using TMS as an internal standard. Mass spectra were recorded on a VG-Micromass $7070 \mathrm{H}$ spectrometer operating at $70 \mathrm{eV}$.

$1 H$-Indole-4-carboxylic acid ethyl ester (2) To the solution of $1 H$-indole-4-carboxylic acid (1) $(0.01 \mathrm{~mol})$ in absolute ethyl alcohol $(15 \mathrm{ml})$, conc. $\mathrm{H}_{2} \mathrm{SO}_{4}(2 \mathrm{ml})$ was added. The mixture was refluxed for $4 \mathrm{~h}$. After completion of the reaction (monitored by TLC), the solvent was removed under reduced pressure and the obtained residue was recrystallized with petroleum ether to get pure $1 \mathrm{H}$-indole-4-carboxylic acid ethyl ester (2).

$1 H$-Indole-4-carboxylic acid hydrazide (3) A mixture of $1 H$-indole-4-carboxylic acid ethyl ester (2) $(0.01 \mathrm{~mol})$ and hydrazine hydrate $(0.025 \mathrm{~mol})$ in ethanol $(20 \mathrm{ml})$ was refluxed for $8 \mathrm{~h}$. After completion of the reaction (monitored by TLC), the mixture was cooled to room temperature and filtered. The crude product was recrystallized from ethanol to give $1 \mathrm{H}$-indole-4carboxylic acid hydrazide (3) in pure form.

5-(1H-Indole-4-yl)-[1,3,4]oxadiazole-2-thiol (4) A mixture of $1 \mathrm{H}$-indole-4-carboxylic acid hydrazide (3) (0.01 mol), potassium hydroxide $(0.02 \mathrm{~mol})$ and carbon disulfide $(0.03 \mathrm{~mol})$ in ethanol $(100 \mathrm{~mL})$ was heated under reflux with stirring for $14 \mathrm{~h}$. The solvent was distilled in vacuo, the residual mass was poured over crushed ice and neutralized the alkaline solution with $10 \%$ hydrochloric acid. The precipitated crude product was filtered, washed with water, dried and recrystallized from ethanol to get the pure compound 5-( $1 H$-indole-4yl)-[1,3,4] oxadiazole-2-thiol (4). 


\section{4-Amino-5-(1H-indol-4-yl)-4H-[1,2,4]-triazole-3-thiol}

(5) To a warm solution of 5-(1H-indole-4-yl)-[1,3,4]oxadiazole-2-thiol (4) $(0.01 \mathrm{~mol})$ in ethanol $(20 \mathrm{~mL})$, $80 \%$ hydrazine hydrate $(0.03 \mathrm{~mol})$ was added drop wise and the reaction mixture was heated under reflux for $7 \mathrm{~h}$. The solvent was distilled off in vacuo, cooled and the solid separated were filtered, washed with cold ethanol and recrystallized from chloroform to give pure compound 4-amino-5-(1H-indol-4-yl)-4H-[1,2,4]triazole-3-thiol (5).

\section{3-(1H-indole-4-yl)-6-aryl-7H-[1,2,4]-triazolo-[3,4-}

b] $[1,3,4]$-thiadiazines (6a-f) A mixture of 4-amino-5(1H-indol-4-yl)-4H-[1,2,4]-triazole-3-thiol (5) $(0.01 \mathrm{~mol})$ and corresponding phenacylbromide $(0.02 \mathrm{~mol})$ in absolute ethanol $(20 \mathrm{~mL})$ was refluxed for $8-10 \mathrm{~h}$. The reaction mixture was concentrated and cooled to room temperature, and the remaining solvent was removed under reduced pressure, then diethyl ether $(25 \mathrm{~mL})$ was added and the reaction mixture was left at $0{ }^{\circ} \mathrm{C}$ for overnight. The precipitated solid was filtered off. The crude product thus obtained was purified by column chromatography on silica gel with hexane-ethyl acetate as eluent to afford pure 3-(1H-indole-4-yl)-6-aryl- $7 \mathrm{H}$ [1,2,4]-triazolo-[3,4-b][1,3,4]-thiadiazines (6a-f).

\section{PHYSICAL AND SPECTRAL DATA}

1H-Indole-4-carboxylic acid ethyl ester (2) Yellow solid; yield 78\%; bp 342-343 ${ }^{\circ} \mathrm{C}$; IR $(\mathrm{KBr}) \mathrm{cm}^{-1}$ : 3212 $(\mathrm{N}-\mathrm{H}), 3024(\mathrm{Ar}-\mathrm{H}), 2962\left(\mathrm{C}-\mathrm{H}, \mathrm{CH}_{3}\right), 1699(\mathrm{C}=\mathrm{N}), 1588$ $(\mathrm{C}=\mathrm{C}) ;{ }^{1} \mathrm{H}-\mathrm{NMR}\left(\mathrm{CDCl}_{3}\right) \delta: 1.24(3 \mathrm{H}, \mathrm{t}, J=5.6 \mathrm{~Hz}$, $\left.\mathrm{CH}_{3}\right), 4.00\left(2 \mathrm{H}, \mathrm{q}, J=5.6 \mathrm{~Hz}, \mathrm{CH}_{2}\right), 7.42(1 \mathrm{H}, \mathrm{d}, J=7.4$ $\mathrm{Hz}, \mathrm{Ar}-\mathrm{H}), 7.39-7.62(3 \mathrm{H}, \mathrm{m}, \mathrm{Ar}-\mathrm{H}), 7.85(1 \mathrm{H}, \mathrm{d}, J=7.4$ $\mathrm{Hz}, \mathrm{Ar}-\mathrm{H}), 11.12(1 \mathrm{H}, \mathrm{s}, \mathrm{NH}) ;{ }^{13} \mathrm{C}-\mathrm{NMR}\left(\mathrm{CDCl}_{3}\right) \delta$ : 13.6, 59.1, 106.3, 112.5, 119.4, 121.7, 122.8, 126.3, 130.4, 132.5, 165.4; MS m/z: $189\left(\mathrm{M}^{+}\right)$; Elemental analysis calculated for $\mathrm{C}_{11} \mathrm{H}_{11} \mathrm{NO}_{2}$ : C-69.83, H-5.86, N7.40, O-16.91. Found: C-67.36, H-5.42, N-7.06, O15.98 .

1H-Indole-4-carboxylic acid hydrazide (3) Brown solid; yield $81 \%$; mp $187-189{ }^{\circ} \mathrm{C}$; IR $(\mathrm{KBr}) \mathrm{cm}^{-1}$ : 3318 $\left(\mathrm{NH}_{2}\right), 3218(\mathrm{~N}-\mathrm{H}), 3065(\mathrm{Ar}-\mathrm{H}), 1645(\mathrm{C}=\mathrm{N}), 1548$ $(\mathrm{C}=\mathrm{C}) ;{ }^{1} \mathrm{H}-\mathrm{NMR}\left(\mathrm{CDCl}_{3}\right) \delta: 5.30\left(2 \mathrm{H}, \mathrm{s}, \mathrm{NH}_{2}\right), 7.38(1 \mathrm{H}$, $\mathrm{d}, J=7.2 \mathrm{~Hz}, \mathrm{Ar}-\mathrm{H}), 7.42-7.64(3 \mathrm{H}, \mathrm{m}, \mathrm{Ar}-\mathrm{H}), 7.70(1 \mathrm{H}$, s, NH), $7.79(1 \mathrm{H}, \mathrm{d}, J=7.2 \mathrm{~Hz}, \mathrm{Ar}-\mathrm{H}), 11.06(1 \mathrm{H}, \mathrm{s}$, $\mathrm{NH}) ;{ }^{13} \mathrm{C}-\mathrm{NMR}\left(\mathrm{CDCl}_{3}\right) \delta$ : 105.7, 114.2, 121.0, 123.7, 124.4, 127.3, 132.7, 135.3, 162.3; MS m/z: $175\left(\mathrm{M}^{+}\right)$; Elemental analysis calculated for $\mathrm{C}_{9} \mathrm{H}_{9} \mathrm{~N}_{3} \mathrm{O}: \mathrm{C}-61.70, \mathrm{H}-$ 5.18, N-23.99, O-9.13. Found: C-60.12, H-4.89, N$22.17, \mathrm{O}-8.89$.

5-(1H-Indole-4-yl)-[1,3,4]oxadiazole-2-thiol (4) Pale yellow solid; yield 74\%; mp $185-187{ }^{\circ} \mathrm{C}$; IR $(\mathrm{KBr}) \mathrm{cm}^{-1}$ : $3236(\mathrm{~N}-\mathrm{H}), 3028$ (Ar-H), $2610(\mathrm{~S}-\mathrm{H}), 1648(\mathrm{C}=\mathrm{N})$, $1559(\mathrm{C}=\mathrm{C}), 1155 ;{ }^{1} \mathrm{H}-\mathrm{NMR}\left(\mathrm{CDCl}_{3}\right) \delta: 3.81(1 \mathrm{H}, \mathrm{s}$, $\mathrm{SH}), 7.35(1 \mathrm{H}, \mathrm{d}, J=7.6 \mathrm{~Hz}, \mathrm{Ar}-\mathrm{H}), 7.41-7.58(3 \mathrm{H}, \mathrm{m}$, Ar-H), $7.79(1 \mathrm{H}, \mathrm{d}, J=7.6 \mathrm{~Hz}, \mathrm{Ar}-\mathrm{H}), 11.24(1 \mathrm{H}, \mathrm{s}$, $\mathrm{NH}) ;{ }^{13} \mathrm{C}-\mathrm{NMR}\left(\mathrm{CDCl}_{3}\right) \delta$ : 102.4, 116.3, 120.7, 123.4, 125.8, 128.4, 136.1, 139.8, 145.6, 158.9; MS m/z: 217 $\left(\mathrm{M}^{+}\right)$; Elemental analysis calculated for $\mathrm{C}_{10} \mathrm{H}_{7} \mathrm{~N}_{3} \mathrm{OS}$ : C55.29, H-3.25, N-19.34, O-7.69, S-14.76. Found: C53.69, H-3.12, N-18.45, O-7.02, S-13.38.

4-Amino-5-(1H-indol-4-yl)-4H-[1,2,4]-triazole-3-thiol (5) White solid; yield $72 \%$; mp $147-149{ }^{\circ} \mathrm{C}$; IR (KBr) (c) 2011, JDDT. All Rights Reserved JDDTAO $\mathrm{cm}^{-1}: 3248(\mathrm{~N}-\mathrm{H}), 3018(\mathrm{Ar}-\mathrm{H}), 2648(\mathrm{~S}-\mathrm{H}), 1662$ $(\mathrm{C}=\mathrm{N}), 1552(\mathrm{C}=\mathrm{C}) ;{ }^{1} \mathrm{H}-\mathrm{NMR}\left(\mathrm{CDCl}_{3}\right) \delta: 3.65(1 \mathrm{H}, \mathrm{s}$, $\mathrm{SH}), 3.85\left(2 \mathrm{H}, \mathrm{s}, \mathrm{NH}_{2}\right), 7.37(1 \mathrm{H}, \mathrm{d}, J=7.3 \mathrm{~Hz}, \mathrm{Ar}-\mathrm{H})$, 7.45-7.74 (3H, m, Ar-H), $7.80(1 \mathrm{H}, \mathrm{d}, J=7.3 \mathrm{~Hz}, \mathrm{Ar}-\mathrm{H})$, $11.21(1 \mathrm{H}, \mathrm{s}, \mathrm{NH}) ;{ }^{13} \mathrm{C}-\mathrm{NMR}\left(\mathrm{CDCl}_{3}\right) \delta: 105.6,110.8$, 116.7, 119.4, 127.6, 129.4, 132.4, 137.6, 142.3, 152.7; MS $m / z: 231\left(\mathrm{M}^{+}\right)$; Elemental analysis calculated for $\mathrm{C}_{10} \mathrm{H}_{9} \mathrm{~N}_{5} \mathrm{~S}$ : C-51.93, H-3.92, N-30.28, S-13.86. Found: C-50.12, H-3.45, N-29.65, S-12.98.

3-(1H-Indole-4-yl)-6-phenyl-7H-[1,2,4]-triazolo-[3,4b] $1,3,4]$-thiadiazine (6a) Pink solid; yield 75\%; mp 158-160 ${ }^{\circ} \mathrm{C}$; IR (KBr) cm ${ }^{-1}: 3245(\mathrm{~N}-\mathrm{H}), 3018(\mathrm{Ar}-\mathrm{H})$, $2965\left(\mathrm{C}-\mathrm{H}, \mathrm{CH}_{2}\right), 1638(\mathrm{C}=\mathrm{N}), 1548(\mathrm{C}=\mathrm{C}) ;{ }^{1} \mathrm{H}-\mathrm{NMR}$ $\left(\mathrm{CDCl}_{3}\right) \delta: 1.86\left(2 \mathrm{H}, \mathrm{s}, \mathrm{CH}_{2}\right), 7.32(1 \mathrm{H}, \mathrm{d}, J=6.8 \mathrm{~Hz}$, Ar-H), 7.41-7.74 (8H, m, Ar-H), $7.79(1 \mathrm{H}, \mathrm{d}, J=6.8 \mathrm{~Hz}$, $\mathrm{Ar}-\mathrm{H}), 10.98(1 \mathrm{H}, \mathrm{s}, \mathrm{NH}) ;{ }^{13} \mathrm{C}-\mathrm{NMR}\left(\mathrm{CDCl}_{3}\right) \delta: 33.7$, $104.3,116.7,121.0,125.4,126.3,128.7$ (2), 129.7, $130.2,131.7$ (2), 132.7, 133.1, 138.4, 144.2, 148.7, 162.3; MS $m / z$ : $331\left(\mathrm{M}^{+}\right)$; Elemental analysis calculated for $\mathrm{C}_{18} \mathrm{H}_{13} \mathrm{~N}_{5} \mathrm{~S}$ : C-65.24, $\mathrm{H}-3.95, \quad \mathrm{~N}-21.13, \quad \mathrm{~S}-9.68$. Found: C-63.25, H-3.64, N-20.28, S-8.95.

3-(1H-Indole-4-yl)-6-(4-methoxy-phenyl)-7H-[1,2,4]triazolo-[3,4-b][1,3,4]-thiadiazine (6b) Pink solid; yield $80 \%$; mp 162-164 ${ }^{\circ} \mathrm{C}$; IR (KBr) cm ${ }^{-1}: 3239(\mathrm{~N}-\mathrm{H}), 3024$ (Ar-H), $2960\left(\mathrm{C}-\mathrm{H}, \mathrm{CH}_{2}\right), 1642(\mathrm{C}=\mathrm{N}), 1568(\mathrm{C}=\mathrm{C})$, $1145(\mathrm{C}-\mathrm{O}) ;{ }^{1} \mathrm{H}-\mathrm{NMR}\left(\mathrm{CDCl}_{3}\right) \delta: 1.74\left(2 \mathrm{H}, \mathrm{s}, \mathrm{CH}_{2}\right), 1.24$ $\left(3 \mathrm{H}, \mathrm{s}, \mathrm{CH}_{3}\right), 7.29(1 \mathrm{H}, \mathrm{d}, J=7.0 \mathrm{~Hz}, \mathrm{Ar}-\mathrm{H}), 7.32(2 \mathrm{H}, \mathrm{d}$, $J=7.2 \mathrm{~Hz}, \mathrm{Ar}-\mathrm{H}), 7.40$ (2H, d, $J=7.2 \mathrm{~Hz}, \mathrm{Ar}-\mathrm{H}), 7.42-$ $7.81(3 \mathrm{H}, \mathrm{m}, \mathrm{Ar}-\mathrm{H}), 7.85(1 \mathrm{H}, \mathrm{d}, J=7.0 \mathrm{~Hz}, \mathrm{Ar}-\mathrm{H})$, $10.84(1 \mathrm{H}, \mathrm{s}, \mathrm{NH}) ;{ }^{13} \mathrm{C}-\mathrm{NMR}\left(\mathrm{CDCl}_{3}\right) \delta: 30.3,44.7$, $106.7,118.4,123.4,126.3,128.7,129.6$ (2), 130.7, 132.7, 133.2 (2), 134.1, 135.2, 137.4, 146.7, 149.5, 163.1; MS m/z: $361\left(\mathrm{M}^{+}\right)$; Elemental analysis calculated for $\mathrm{C}_{19} \mathrm{H}_{15} \mathrm{~N}_{5} \mathrm{OS}$ : C-63.14, H-4.18, N-19.38, O-4.43, S8.87. Found: C-61.23, H-3.84, N-18.28, O-4.12, S-7.84.

\section{3-(1H-Indole-4-yl)-6-(4-chloro-phenyl)-7H-[1,2,4]-} triazolo-[3,4-b][1,3,4]-thiadiazine (6c) Yellow solid; yield $82 \%$; mp $126-128{ }^{\circ} \mathrm{C}$; IR $(\mathrm{KBr}) \mathrm{cm}^{-1}$ : $3240(\mathrm{~N}-\mathrm{H})$, 3028 (Ar-H), $2958\left(\mathrm{C}-\mathrm{H}, \mathrm{CH}_{2}\right), 1654(\mathrm{C}=\mathrm{N}), 1595$ $(\mathrm{C}=\mathrm{C}) ;{ }^{1} \mathrm{H}-\mathrm{NMR}\left(\mathrm{CDCl}_{3}\right) \delta: 1.68\left(2 \mathrm{H}, \mathrm{s}, \mathrm{CH}_{2}\right), 7.26(1 \mathrm{H}$, $\mathrm{d}, J=7.4 \mathrm{~Hz}, \mathrm{Ar}-\mathrm{H}), 7.30(2 \mathrm{H}, \mathrm{d}, J=7.0 \mathrm{~Hz}, \mathrm{Ar}-\mathrm{H}), 7.38$ $(2 \mathrm{H}, \mathrm{d}, J=7.0 \mathrm{~Hz}, \mathrm{Ar}-\mathrm{H}), 7.46-7.76(3 \mathrm{H}, \mathrm{m}, \mathrm{Ar}-\mathrm{H}), 7.79$ $(1 \mathrm{H}, \mathrm{d}, J=7.4 \mathrm{~Hz}, \mathrm{Ar}-\mathrm{H}), 10.92(1 \mathrm{H}, \mathrm{s}, \mathrm{NH}) ;{ }^{13} \mathrm{C}-\mathrm{NMR}$ $\left(\mathrm{CDCl}_{3}\right) \delta: 32.3,104.2,116.2,119.7,122.0,124.2,125.3$ (2), 128.4, 136.3, 139.2 (2), 140.2, 141.0, 142.7, 148.7, 150.2, 165.7; MS m/z: $365\left(\mathrm{M}^{+}\right)$; Elemental analysis calculated for $\mathrm{C}_{18} \mathrm{H}_{12} \mathrm{ClN}_{5} \mathrm{~S}$ : C-59.09, H-3.31, Cl-9.69, N-19.14, S-8.76. Found: C-57.16, H-3.14, Cl-8.84, N$18.56, \mathrm{~S}-7.48$.

3-(1H-Indole-4-yl)-6-(4-bromo-phenyl)-7H-[1,2,4]triazolo-[3,4-b][1,3,4]-thiadiazine (6d) Brown solid; yield $81 \%$; mp $132-134{ }^{\circ} \mathrm{C}$; IR $(\mathrm{KBr}) \mathrm{cm}^{-1}$ : $3258(\mathrm{~N}-\mathrm{H})$, $3032(\mathrm{Ar}-\mathrm{H}), 2962\left(\mathrm{C}-\mathrm{H}, \mathrm{CH}_{2}\right), 1664(\mathrm{C}=\mathrm{N}), 1565$ $(\mathrm{C}=\mathrm{C}) ;{ }^{1} \mathrm{H}-\mathrm{NMR}\left(\mathrm{CDCl}_{3}\right) \delta: 1.54\left(2 \mathrm{H}, \mathrm{s}, \mathrm{CH}_{2}\right), 7.16(1 \mathrm{H}$, $\mathrm{d}, J=7.3 \mathrm{~Hz}, \mathrm{Ar}-\mathrm{H}), 7.26(2 \mathrm{H}, \mathrm{d}, J=7.3 \mathrm{~Hz}, \mathrm{Ar}-\mathrm{H}), 7.34$ (2H, d, $J=7.5 \mathrm{~Hz}$, Ar-H), 7.40-7.68 (3H, m, Ar-H), 7.72 $(1 \mathrm{H}, \mathrm{d}, J=7.5 \mathrm{~Hz}, \mathrm{Ar}-\mathrm{H}), 10.86(1 \mathrm{H}, \mathrm{s}, \mathrm{NH}) ;{ }^{13} \mathrm{C}-\mathrm{NMR}$ $\left(\mathrm{CDCl}_{3}\right) \delta: 36.3,107.4,112.0,115.7,118.6,120.8,122.3$ (2), 124.7, 132.6, 134.8 (2), 138.7, 140.2, 145.7, 146.3, 152.7, 163.8; MS m/z: $410\left(\mathrm{M}^{+}\right)$; Elemental analysis calculated for $\mathrm{C}_{18} \mathrm{H}_{12} \mathrm{BrN}_{5} \mathrm{~S}$ : C-52.69, H-2.95, Br-19.47, 
Mekala et al

Journal of Drug Delivery \& Therapeutics; 2014, 4(2), 43-46

N-17.07, S-7.89. Found: C-50.36, H-2.65, Br-18.84, N16.47, S-9.89.

\section{3-(1H-Indole-4-yl)-6-(4-nitro-phenyl)-7H-[1,2,4]-} triazolo- $[3,4-b][1,3,4]$-thiadiazine $(\mathbf{6 e})$ White solid; yield $79 \%$; mp $154-156{ }^{\circ} \mathrm{C}$; IR $(\mathrm{KBr}) \mathrm{cm}^{-1}: 3262(\mathrm{~N}-\mathrm{H})$, 3027 (Ar-H), 2945 (C-H, CH $), 1652(\mathrm{C}=\mathrm{N}), 1556$ $(\mathrm{C}=\mathrm{C}) ;{ }^{1} \mathrm{H}-\mathrm{NMR}\left(\mathrm{CDCl}_{3}\right) \delta: 1.58\left(2 \mathrm{H}, \mathrm{s}, \mathrm{CH}_{2}\right), 7.26(1 \mathrm{H}$, $\mathrm{d}, J=6.8 \mathrm{~Hz}, \mathrm{Ar}-\mathrm{H}), 7.31(2 \mathrm{H}, \mathrm{d}, J=6.8 \mathrm{~Hz}, \mathrm{Ar}-\mathrm{H}), 7.38$ (2H, d, J = 7.2 Hz, Ar-H), 7.45-7.72 (3H, m, Ar-H), 7.78 $(1 \mathrm{H}, \mathrm{d}, J=7.2 \mathrm{~Hz}, \mathrm{Ar}-\mathrm{H}), 11.08(1 \mathrm{H}, \mathrm{s}, \mathrm{NH}) ;{ }^{13} \mathrm{C}-\mathrm{NMR}$ $\left(\mathrm{CDCl}_{3}\right) \delta: 33.7,108.9,114.7,117.4,119.6,122.3,124.7$ (2), 126.8, 133.7, 135.8 (2), 139.7, 142.4, 148.7, 149.6, 153.7, 164.9; MS m/z: $376\left(\mathrm{M}^{+}\right)$; Elemental analysis calculated for $\mathrm{C}_{18} \mathrm{H}_{12} \mathrm{~N}_{6} \mathrm{O}_{2} \mathrm{~S}$ : C-57.44, H-3.21, N-22.33, O-8.50, S-8.52. Found: C-55.98, H-3.12, N-21.28, O7.87, S-7.95.

\section{REFERENCES:}

1) Demirbas N, Karaoglu SA, Demirbas A, Celik E, Arkivoc. 2005, (i), 75-91.

2) Marina K, Anastasia M, Panagiotis M, Nicole P, Spyroula P-G, Christophe P, Myriam W, Erik De C, Il Farmaco 2002, 57, 253-257.

3) Xiao-Wen S, Zhang Yan, Zhang Zi-Yi, Wang Qin, Wang ShuFang, Indian J Chem. 1999, 38B, 380-383.

4) Udupi RH, Suresh GV, Setty SR, Bhat AR, J Indian Chem Soc. 2000, 77, 302-304.

5) Nizamuddin, Gupta M, Khan MH, Srivastava MK, J Sci Ind Res. 1999, 58, 538-542.
3-(1H-Indole-4-yl)-6-(4-hydroxy-phenyl)-7H-[1,2,4]triazolo-[3,4-b][1,3,4]-thiadiazine (6f) Pale solid; yield 77\%; mp 139-141 ${ }^{\circ} \mathrm{C}$; IR (KBr) cm ${ }^{-1}: 3256(\mathrm{~N}-\mathrm{H}), 3042$ (Ar-H), $2938\left(\mathrm{C}-\mathrm{H}, \mathrm{CH}_{2}\right), 1665(\mathrm{C}=\mathrm{N}), 1548(\mathrm{C}=\mathrm{C}) ;{ }^{1} \mathrm{H}-$ NMR $\left(\mathrm{CDCl}_{3}\right) \delta: 1.46\left(2 \mathrm{H}, \mathrm{s}, \mathrm{CH}_{2}\right), 5.02(1 \mathrm{H}, \mathrm{s}, \mathrm{OH})$, $7.22(1 \mathrm{H}, \mathrm{d}, J=7.0 \mathrm{~Hz}, \mathrm{Ar}-\mathrm{H}), 7.35(2 \mathrm{H}, \mathrm{d}, J=7.0 \mathrm{~Hz}$, Ar-H), 7.40 (2H, d, $J=7.4 \mathrm{~Hz}, \mathrm{Ar}-\mathrm{H}), 7.48-7.78(3 \mathrm{H}, \mathrm{m}$, Ar-H), $7.84(1 \mathrm{H}, \mathrm{d}, J=7.4 \mathrm{~Hz}, \mathrm{Ar}-\mathrm{H}), 11.10(1 \mathrm{H}, \mathrm{s}$, $\mathrm{NH}) ;{ }^{13} \mathrm{C}-\mathrm{NMR}\left(\mathrm{CDCl}_{3}\right) \delta: 30.3,112.8,115.4,116.3$, 121.4, 123.7, 126.8 (2), 128.7, 135.7, 138.7 (2), 140.2, 143.6, 145.7, 147.8, 151.6, 165.6; MS m/z: $347\left(\mathrm{M}^{+}\right)$; Elemental analysis calculated for $\mathrm{C}_{18} \mathrm{H}_{13} \mathrm{~N}_{5} \mathrm{OS}$ : C-62.23, H-3.77, N-20.16, O-4.61, S-9.23. Found: C-60.54, H3.42, N-19.65, O-4.21, S-8.85.

6) Invidiata FP, Simoni D, Scintu F, Pinna N, Farmaco. 1996, 51, 659-664.

7) Tozkoparan B, Gokhan N, Aktay G, Yesilada E, Ertan M, Eur J Med Chem. 2000, 35, 743-750.

8) C.A. Winter, E.A. Risley, G.N. Nus Carrageenin-induced edema in hind paw of the rat as assay for antiinflammatory drugs, Proc Soc Exp Biol Med. 111, 1962, 544.

9) National Committee for Clinical Laboratory Standards (NCCLS). Standard methods for dilution antimicrobial susceptibility tests for bacteria, which grows aerobically. Nat. Comm. Lab. Stands, Villanova, 1982, 242. 